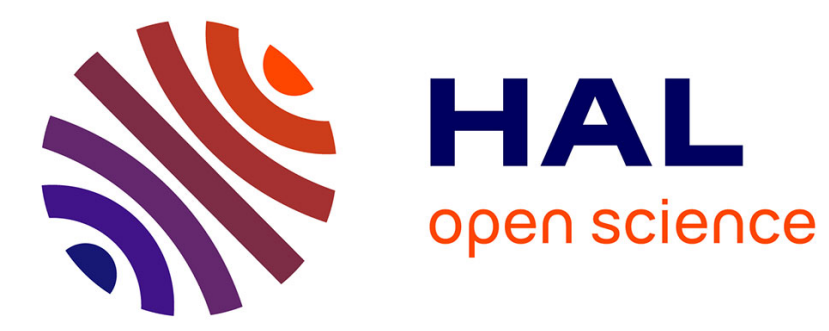

\title{
The repertoire of seizure onset patterns in human focal epilepsies: Determinants and prognostic values
}

Stanislas Lagarde, Sinziana Buzori, Agnès Trebuchon, Romain Carron, Didier Scavarda, Mathieu Milh, Aileen Mcgonigal, Fabrice Bartolomei

\section{- To cite this version:}

Stanislas Lagarde, Sinziana Buzori, Agnès Trebuchon, Romain Carron, Didier Scavarda, et al.. The repertoire of seizure onset patterns in human focal epilepsies: Determinants and prognostic values. Epilepsia, 2019, 60 (1), pp.85-95. 10.1111/epi.14604 . hal-03563037

\section{HAL Id: hal-03563037 https://hal.science/hal-03563037}

Submitted on 9 Feb 2022

HAL is a multi-disciplinary open access archive for the deposit and dissemination of scientific research documents, whether they are published or not. The documents may come from teaching and research institutions in France or abroad, or from public or private research centers.
L'archive ouverte pluridisciplinaire HAL, est destinée au dépôt et à la diffusion de documents scientifiques de niveau recherche, publiés ou non, émanant des établissements d'enseignement et de recherche français ou étrangers, des laboratoires publics ou privés. 


\section{THE REPERTOIRE OF SEIZURE ONSET PATTERNS IN HUMAN FOCAL EPILEPSIES: \\ DETERMINANTS AND PROGNOSTIC VALUES}

\section{Authors:}

Stanislas Lagarde * 1, Sinziana Buzori * 1, Agnès Trebuchon 1, Romain Carron 2, Didier Scavarda 3 , Mathieu Milh ${ }^{4}$, Aileen McGonigal ${ }^{1}$, Fabrice Bartolomei ${ }^{1}$

*These authors contributed equally to this work

\section{Affiliations :}

${ }^{1}$ Aix Marseille Univ, APHM, INSERM, INS, Inst Neurosci Syst, Timone Hospital, Epileptology Department, Marseille, France ;

${ }^{2}$ Aix Marseille Univ, APHM, INSERM, INS, Inst Neurosci Syst, Timone Hospital, Functional and Stereotactic Neurosurgery, Marseille, France ;

${ }^{3}$ APHM, Timone Hospital, Pediatric Neurosurgery, Marseille, France;

${ }^{4}$ APHM, Timone Hospital, Pediatric Neurology, Marseille, France;

\section{Corresponding author:}

Prof Fabrice Bartolomei, MD, PhD

Address: Service d'Epileptologie et Rythmologie Cérébrale, CHU Timone - 264 Rue St Pierre 13005-Marseille, France

Phone: +33491385833; Fax: +33491385826;

Email: fabrice.bartolomei@ap-hm.fr

Key Words: Epilepsy; Focal Drug-Resistant Epilepsy; SEEG; Seizure-Onset; Epileptogenic zone; Epilepsy Surgery

Number of Text Pages: 14; Number of words: 3971; Number of figures: 6; Number of References: 50 


\section{SUMMARY}

Objective: In this study, we seek to analyze the determinants of the intracranial EEG seizure onset pattern and the impact of the seizure onset pattern in predicting postsurgical seizure outcome.

Methods: To this end, we analyzed 820 seizures from 252 consecutive patients explored by SEEG (total of 2148 electrodes) including various forms of focal refractory epilepsies. We used a reproducible method combining visual and time-frequency analyses.

Results: We described eight seizure onset patterns: low-voltage fast activity (LVFA), preictal spiking followed by LVFA, burst of poly-spikes followed by LVFA, slow wave/DC shift followed by LVFA, theta/alpha sharp waves, beta sharp waves, rhythmic spikes/spike-waves and delta-brush. LVFA occurred in $79 \%$ of patients. The seizureonset pattern was significantly associated with: 1) underlying etiology (burst of polyspikes followed by LVFA with the presence of a focal cortical dysplasia, LVFA with malformation of cortical development, post-vascular and undetermined epilepsies); 2) spatial organization of the epileptogenic zone (burst of poly-spikes followed by LVFA with focal organization, slow wave/DC shift followed by LVFA with network organization); 3) post-surgical seizure outcome (better outcome when LVFA present).

Significance: This study demonstrates that the main determinants of the seizure onset pattern are the underlying etiology and the spatial organization of the epileptogenic zone. Concerning the post-surgical seizure outcome, the main determinant factor is the spatial organization of the epileptogenic zone, but the seizure onset pattern plays also a role, conferring better prognosis when LVFA was present.

Key words: Epilepsy; Focal Drug-resistant Epilepsy; SEEG; Seizure-onset; Epileptogenic zone; Epilepsy Surgery 


\section{KEY POINT BOX}

1. The main determinants of the seizure onset pattern are the underlying etiology and the spatial organization of the epileptogenic zone.

2. The main determinant factor of post-surgical seizure outcome is the spatial organization of the epileptogenic zone (focal vs network).

3. The seizure onset pattern plays also a role, conferring better prognosis when LVFA was present. 


\section{MANUSCRIPT}

\section{INTRODUCTION}

Resective surgery, when possible, is the best treatment for focal pharmacoresistant epilepsy. Optimal determination of the epileptogenic zone (EZ) often requires intracranial recordings such as stereo-EEG (SEEG), especially in cases of negative magnetic resonance imaging (MRI), widespread epilepsies, and proximity of eloquent areas $^{1}$. SEEG interpretation ${ }^{2}$ is crucially based on analysis of seizure dynamics including seizure onset pattern (SOP) and the spatial extent of the discharge (EZ organization). A limited number of distinct SOP have been described ${ }^{3-5}$, suggesting a limited repertoire of interictal to ictal transitions at the macroscopic scale.

From a pathophysiological point of view, identifying SOP is of utmost importance for understanding the mechanisms of interictal to ictal transition. Animal studies have demonstrated that distinct neuronal populations are involved in the genesis of fast activity and rhythmic paroxysms ${ }^{6}$. In humans, pre-ictal synchrony among EZ structures appears linked to the SOP7,8. Therefore, the study of SOP could improve our understanding of seizure generation and hopefully lead to new therapeutic targets. For instance, in closed-loop strategies, detection algorithms depend on proper understanding of the mechanisms underpinning the transition between the interictal and ictal state in order to efficiently trigger stimulation and abort seizures.

Previous studies have reported distinct SOP in mesio-temporal and neocortical epilepsies $^{3}$. The SOP was also correlated with the degree of mesio-temporal atrophy ${ }^{4,9-}$ 14. Of note, most previous reports did not find a clear relationship between lesion type and SOP ${ }^{4}$. However, we recently suggested that some SOP are linked to histopathology in patients with malformations of cortical development (MCD) ${ }^{5}$. Some studies have also 
suggested different SOP according to focal or regional organization of the EZ ${ }^{4,15}$. The value of SOP in predicting post-surgical seizure outcome has been also investigated, suggesting better outcome in the presence of low-voltage fast activity 3,5 .

Nevertheless, some aspects of previous literature preclude a global conclusion about the relation between SOP, clinical variables and surgical outcome. Firstly, most studies have used different methods of intracranial recordings (grids, depth or mixed electrodes) leading to limited comparability. Secondly, most have focused on temporal epilepsies, thus not covering the whole spectrum of surgical epilepsies. Thirdly, relations between SOP and other clinical variables (such as EZ localization, etiology, presence of MRI lesion and EZ organization) have not been investigated using homogenous methodology in a large series. Finally, the association between SOP and post-surgical seizure outcome has not been studied with multivariate analysis taking into account confounding variables (such as EZ extension).

In the present study, we applied our previously published methodology ${ }^{5}$ in order to reproducibly analyze the SOP of 820 seizures in 252 consecutive patients explored by SEEG. We sought to study predictors of the SOP, and the impact of SOP in predicting post-surgical seizure outcome.

\section{METHODS}

\section{Patient selection}

We retrospectively analyzed all consecutive patients who underwent stereotacticelectroencephalography (SEEG) in our department from 2000 to 2016 . All patients had detailed presurgical work-up, including medical history, neurologic examination, cerebral MRI, cerebral positron emission tomography (PET) and scalp-EEG recordings. 
In this study, all patients required SEEG as part of usual clinical care (epileptogenic zone (EZ) suspected to be larger than the lesion, and/or overlapping with eloquent cortices, and/or extra temporal regions suspected to be involved, and/or when bilateral involvement suspected, and/or lack of MRI lesions ${ }^{16}$ ). The placement of electrodes was selected, in each patient, based on available non-invasive information providing hypotheses about the localization of the EZ. We excluded patients without spontaneous seizures (apart from stimulation) and/or with sub-optimal ictal recording (e.g. deficient contact or electrode, absence of SEEG ictal discharge).

For patients who were operated on, an expert neuropathologist reviewed histological data and established the pathological diagnosis according to current ILAE and WHO classifications ${ }^{17-19}$. The institutional review board of the French Institute of Health (IRB15226) approved this study and patients' written consent was obtained.

\section{SEEG recordings}

Recordings were performed using intracerebral multiple contact electrodes (10-15 contacts with length $2 \mathrm{~mm}$, diameter $0.8 \mathrm{~mm}$, and $1.5 \mathrm{~mm}$ apart; for details see ${ }^{5}$ ). Signals were recorded on a 128 or 256-channel Natus system and sampled at 256, 512, or $1,024 \mathrm{~Hz}$, depending on the period of recording, and recorded on a hard disk (16 bits/ sample) using no digital filter. Two hardware filters were present in the acquisition procedure: a high-pass filter (cut-off frequency equal to $0.16 \mathrm{~Hz}$ at $-3 \mathrm{~dB}$ ), and an antialiasing low-pass filter (cut-off frequency equal to $97 \mathrm{~Hz}$ at $256 \mathrm{~Hz}, 170 \mathrm{~Hz}$ at $512 \mathrm{~Hz}$, or $340 \mathrm{~Hz}$ at $1,024 \mathrm{~Hz})$.

\section{Seizure-onset pattern analysis}


Seizure-onset pattern analysis was carried out according to the same methodology as our previous study ${ }^{5}$ The main points are: all seizures of patients were analyzed while blinded to clinical data by two independent epileptologists (SL and SB), and discrepancies were resolved by a senior epileptologist (FB); visual analysis was performed without software filters, and spectral analysis was performed using Amadeus software (LTSI Rennes). The seizure onset was defined as the first alteration of SEEG signal in the context of a sustained rhythmic discharge associated with clinical signs (exclusion of uniquely electrographic seizure). Patients with seizures in which clinical manifestations preceded electrographic onset were excluded from the analysis. Seizureonset SEEG patterns were assessed on the earliest involved electrodes (first 5 seconds).

\section{Clinical Variables}

We defined the EZ using visual analysis complemented by a quantitative method (epileptogenicity index, for detail see ${ }^{20}$ ). Location of electrodes was defined by visual analysis of pre-SEEG MRI fused with the post-operative CT scan performed less than $24 \mathrm{~h}$ after electrode implantation 21 . We classified epilepsies into 12 sub-groups: mesial temporal; lateral temporal; mesio-lateral temporal; temporal plus (temporal and frontal and/or parietal and/or occipital and/or insular, bitemporal); prefrontal; those involving "motor systems" (central, premotor, or both); frontal plus (frontal and temporal and/or parietal and/or insular, bifrontal), operculo-insular; parietal; parietal plus (parietofrontal); occipital; occipital plus (occipital and temporal and/or parietal) $22-25$.

Extension of the EZ was evaluated as the number of distinct brain regions (further referred to as "sub-lobes") involved (defined by high epileptogenicity index $(\geq 0.4)$ or by visual analysis for the cases with lower frequency patterns (structures involved within the first five seconds)). We distinguished the following sub-lobes: mesial temporal, 
lateral temporal, insular, orbito-frontal, mesial prefrontal, lateral prefrontal, premotor, central (pre- and post-central), opercula (frontal and rolandic), mesial parietal, lateral parietal, mesial occipital and lateral occipital. Focal organization corresponded to a situation where only one sub-lobe was involved during the first five seconds of seizure; in other cases the EZ was considered as network-organized.

Definition of the etiology took into account a combination of factors including clinical history, MRI and histological findings. Epilepsy etiology fell into nine sub-groups: malformations of cortical development (focal cortical dysplasia, heterotopia, hemimegalencephaly and tuber), neurodevelopmental tumors (dysembryoplastic and angiocentric neuro-epithelial tumors, ganglioglioma), post-vascular (mainly neonatal stroke), hippocampal sclerosis, post-infectious (mainly encephalitis but also abscess), cavernoma, post-head trauma, others (two patients with Rasmussen's encephalitis and one with hypothalamic hamartoma), and undetermined for the remaining patients (no history of head trauma neither stroke nor infection of CNS, normal MRI, and/or normal or none specific histology such as gliosis).

Surgical outcome was assessed regularly during post-operative follow-up and rated according to Engel's classification ${ }^{26}$. For analysis, we used the surgical outcome score at the latest available follow-up (with exclusion of patients with less than one year of follow-up).

\section{Statistical Analysis}

For statistical analyses, we defined one SOP per patient. For the patients with distinct SOP between seizures (only eight patients in this series), we used the most prevalent pattern. For statistical analyses, we used only categories with more than $2 \%$ of 
prevalence and for the SOP we grouped the two less prevalent patterns. The KruskalWallis rank- sum test was performed to analyze numerical variables, and Fisher's twotailed exact test was used to analyze nominal variables. To achieve our main objectives with limited effect of confounding factors and no inflation of the type-1 (alpha)errorrisk, we used multiple logistic regression analyses. We included in these analyses the previous factors with a p-value $\leq 0.05$ on univariate analyses. We fitted one logistic regression analysis to test the predictive factors of the SOP, and another logistic regression analysis to test the predictive factors of postsurgical outcome. In these analyses, a p-value $\leq 0.05$ was considered to be statistically significant. Statistical tests were performed with the software IBM SPSS Statistics for Mac, version 22.

\section{RESULTS}

\section{Population}

We reviewed two hundred and sixty-five patients, of which 13 were excluded; 252 patients (136 women, 116 men) were selected for this study. A total of 2428 electrodes were implanted in these patients (mean: 11.7 electrodes per patient, SD: 2.6), including $54.5 \%$ bilateral implantations and a mean of 3.4 sampled lobes per patient (SD: 1.1 ). Frontal lobe was sampled in $81.7 \%$ of patients, temporal lobe in $80.4 \%$, parietal lobe in $70.1 \%$, occipital lobe in $21.9 \%$ and insula in $18.3 \%$ (patient features are summarized in supporting information).

The mean number of seizures per patient was 3.3 with a total of 820 seizures analyzed. The mean age at epilepsy onset was 10 years (SD: 9,3); the mean age at SEEG was 26.3 years (SD: 13.8) and the mean epilepsy duration before SEEG was 16.1 years (SD: 10.4). One hundred and ninety-seven patients (78.2\%) had a visible lesion on MRI and 55 
(21.8\%) were considered to be MRI-negative. Fifteen patients (6\%) had had previous surgery. Twenty-eight patients $(11,1 \%)$ had a family history of epilepsy.

The organization of the EZ was focal in 102 patients (40.5\%) and network-organized in 150 patients (59.5\%). The mean number of distinct structures involved was three (SD: $1,7)$.

Concerning the type of epilepsy, we found by decreasing order: 50 temporal plus (19.8\%), 38 mesial temporal (15.1\%), 37 prefrontal (14.7\%), 34 "motor system" (13.5\%), 20 occipital plus (7.9\%), 14 parietal (5.6\%), 13 lateral temporal (5.2\%), 12 operculo-insular (4.8\%), 11 frontal plus (4.4\%), nine mesio-lateral temporal (3.6\%), eight parietal plus (3.2\%), six occipital (2.4\%).

Epilepsy etiology was, in decreasing order: malformation of cortical development in 100 patients (39.7\%), undetermined in 65 patients (25.8\%), neurodevelopmental tumors in 24 patients (9.5\%), post-vascular in 22 patients (8.7\%), hippocampal sclerosis in 17 patients (6.7\%), post-infectious in 9 patients (3.6\%), cavernoma in 6 patients $(2.4 \%)$, traumatic brain injury in 6 patients $(2.4 \%)$, others for 3 patients $(1.2 \%$ : 2 Rasmussen encephalitis and 1 hypothalamic hamartoma)

One hundred and fifty patients (59.5\%) were finally operated on after SEEG (reasons for absence of surgery were: bilateral, multifocal or too extensive EZ organization, EZ overlapping with eloquent cortex, or patient refusal). For 7 patients, insufficient postsurgical follow-up data was available, so for the remaining with a mean follow-up duration of 69.6 months (SD: 37.7): 77 were Engel Class I (53.8\%), 14 Engel Class II (9.8\%), 18 Engel Class III (12.6\%), and 34 Engel Class IV (23.8\%).

\section{Seizure Onset Pattern Prevalence}


Figure 1 shows the eight different SOPs:

A. Low-voltage fast activity (LVFA), with rhythmic fast oscillations $(>14 \mathrm{~Hz}$ ) of low amplitude (<30 microV) starting without initial apparent changes;

B. Preictal spiking with rhythmic spikes of low frequency (classically equal or under $3 \mathrm{~Hz}$ ), high amplitude and prolonged duration (more than 5 seconds, median of 20 s) followed by LVFA (usually of lower frequency than isolated LVFA);

C. Burst of polyspikes of high frequency (typically above $12 \mathrm{~Hz}$ ) with high amplitude and short duration (under 5 seconds) followed by LVFA;

D. Slow wave or baseline shift (equal to DC shift) followed by LVFA;

E. Rhythmic spikes or spike-waves, of low frequency (usually above $6 \mathrm{~Hz}$ and constantly under $14 \mathrm{~Hz}$ ) and with high amplitude;

F. Sharp theta/alpha activity with sinusoidal activity of low frequency (lower than LVFA, median of $9.5 \mathrm{~Hz}$ ), median initial amplitude (higher than LVFA) and progressive increasing amplitude;

G. Sharp beta activity with sinusoid activity of beta-band frequency (lower than LVFA), median initial amplitude (higher than LVFA) and progressive increasing amplitude;

H. Delta-brush, with burst of low amplitude rapid activity (within gamma frequency bands) superimposed upon low frequency (delta) sinusoidal activity.

Overall, 53 patients (21\%) did not present LVFA at seizure onset. More specifically, the prevalence of the different SOP was the following (Figure 2): low-voltage fast activity: 115 (45.6 \%); slow-wave prior to LVFA: 39 (15.5\%); rhythmic slow spikes: 30 (11.9\%); pre-ictal spiking prior to LVFA: 28 (11.1\%); sharp theta/alpha: 17 (6.7\%); burst of polyspikes: 15 (6\%); sharp beta: six (2.4\%); delta-brush: two (0.8\%). 


\section{Predictors of Seizure Onset Pattern}

Figure 3 represents SOP according to epilepsy etiology. We observed some correspondence between type of SOP and etiology: bursts of polyspikes were only seen in MCD; pre-ictal spiking was not observed in post-vascular epilepsy and cavernoma; slow wave or baseline shift were not observed in hippocampal sclerosis and cavernoma; rhythmic slow spikes were not observed in cavernoma; sharp theta/alpha activity was not observed in post-vascular epilepsy and cavernoma. LVFA was found in all etiologies of epilepsy but with an over-representation in MCD, post-vascular epilepsy and those with "unknown" etiology (statistically significant after Bonferroni correction). Globally, there was a significant association between the SOP and the epilepsy etiology $(\mathrm{p}=$ $0.014)$.

Figure 4 represents SOP according to the presence or not of an MRI lesion. All SOP could be observed in both MRI-positive and MRI-negative patients with the exception of bursts of polyspikes, which were only seen in patients with visible MCD on the MRI. There was a significant association with MRI findings $(p=0.007)$ with significant underrepresentation of LVFA and over-representation of slow-wave prior to LVFA in patients with negative MRI (significant after Bonferroni correction, Figure 4).

Figure 5 represents SOP according to type of EZ organization. All SOP were found in both focal and network EZ organization. Nevertheless, there was a significant association with the EZ organization (focal or network; $p=0.022$ ), with an overrepresentation of DC shift / slow-wave prior to LVFA in network EZ organization and an over-representation of bursts of polyspikes prior to LVFA in focal EZ organization (significant after Bonferroni correction, Figure 5). 
Other clinical variables were not significantly associated with SOP: epilepsy type $(\mathrm{p}=0.08)$, epilepsy duration $(\mathrm{p}=0.128)$, history of previous surgery $(\mathrm{p}=0.158)$, age at SEEG ( $p=0.717)$, age at epilepsy onset $(p=0.874)$, and family history of epilepsy $(p=0.87)$.

For multivariate analysis (multinomial logistic regression), we included epilepsy etiology, EZ organization, MRI findings; and we found that epilepsy etiology and EZ organization remained independently and significantly associated with SOP ( $p=0.006$ and 0.022 respectively, Pseudo- $\mathrm{R}^{2}=0.335$ Cox and Snell, 0.349 Nagelkerke) ${ }^{27,28}$.

\section{Predictors of post-surgical prognosis}

We analyzed 143 of the 150 operated patients (excluding seven with missing outcome dataL. Figure 6 represents post-surgical seizure outcome according to SOP. The SOP with the best post-surgical outcome were, in decreasing order of seizure-freedom rate: bursts of polyspikes prior to LVFA (83.3\% seizure-free), LVFA (58.5\% seizure-free), pre-ictal spiking prior to LVFA (57.9 \% seizure-free), slow-wave prior to LVFA (50\% seizurefree), sharp theta/alpha (38.5\% seizure-free), rhythmic slow spikes (30.7\% seizurefree), delta-brush and sharp beta (no patients seizure free). Absence of LVFA was associated with poorer prognosis (only $31 \%$ seizure freedom). Moreover, in patients with SOP including LVFA the presence of a slow-wave prior to the LVFA conferred poorer prognosis, in contrast to the presence of bursts of polyspikes, which indicated excellent outcome. Finally, there was a significant association between post-surgical prognosis (seizure-freedom) and SOP $(\mathrm{p}=0.04)$, with better prognosis in patients with bursts of polyspikes prior to LVFA and less with others (significant after Bonferroni's correction). 
There was also a significant association with the EZ organization $(p=0.001)$, with more favorable prognosis in patients with focal EZ organization. Concerning the association between other clinical variables and post-surgical prognosis: there was no significant association with MRI findings $(\mathrm{p}=0.172)$, type of epilepsy $(\mathrm{p}=0.293)$, age at SEEG $(p=0.297)$, epilepsy duration $(p=0.381)$, history of previous surgery $(p=0.687)$, family history of epilepsy $(\mathrm{p}=0.797)$, age at epilepsy onset $(\mathrm{p}=0.825)$, and epilepsy etiology $(\mathrm{p}=$ 0.981).

After multivariate analysis (multinomial logistic regression including as predictors the SOP and the EZ organization), both SOP and EZ organization remain independently associated with the post-surgical prognosis $(\mathrm{p}=0.023$ and $\mathrm{p}=0.001$ respectively; Pseudo$\mathrm{R}^{2}=0.165$ Cox et Snell, 0.220 Nagelkerke).

\section{DISCUSSION}

Several studies in the past have focused on SOP, but often involved a limited number of patients and were based on heterogeneous recording methods. Direct in situ recording using intracerebral electrodes (SEEG) is probably the best way to capture electrophysiological changes at the onset of seizures. Our study is the first to use a SEEG method in such a large cohort of patients $(n=252)$ and seizures $(n=820)$. We described eight distinct SOPs, separated into three categories: LVFA preceded or not by paroxysmal abnormalities (pre-ictal spiking, burst of polyspikes or slow-wave), rhythmic slow spikes, or sinusoidal sharp activity (ranging from theta to beta frequencyband). Interestingly, we confirmed that $21 \%$ of SOP do not include fast activity. We also did not observe a diffuse electrodecremental pattern, which probably reflects cortical changes recorded at a distance from the EZ, with grids ${ }^{3}$. Our study echoes previous 
literature revealing that SOP could take on various aspects with a limited repertoire of patterns.

We found that epilepsy etiology and EZ organization were the main determinants of SOP and that SOP was associated with post-surgical seizure outcome. SOP and the spatial extent of the initial discharge are two important characteristics of the seizure genesis as recorded during $\mathrm{SEEG}^{2}$.

\section{The etiology of the epilepsy is a significant determinant of the SOP}

It is known that similar lesions could generate distinct SOP and conversely that a SOP could be generated by distinct lesions 4,5. Notably, we demonstrated an association between epilepsy etiology and SOP independently of other clinical covariables. We showed that bursts of polyspikes prior to LVFA were only found in focal cortical dysplasia (FCD), and that LVFA was over-represented in post-vascular epilepsy, MCD and epilepsy of "undetermined" etiology. In our previous study, we have shown bursts of polyspikes to exist in FCD but not in neurodevelopmental tumours 5 . This study extended this result, showing that this SOP appears specific for FCD even in comparison with other etiologies, and could argue for a particular physiopathological mechanism involved in FCD. However, most studies focusing on neocortical epilepsies did not find correlation between SOP and histological substrates ${ }^{3}$. This is probably due to a limited number of patients, and even to the lack of etiological analysis.

Animal experimental studies indicate that distinct neurochemical systems drive distinct SOP6. Injection of a GABA-A agonist (kainite and 4-aminopyridine) triggers LVFA whereas the injection of NMDA agonist (bicuculline) or $\mathrm{GABA}_{\mathrm{A}}$ antagonist (picrotoxin) triggers a pre-ictal spiking pattern ${ }^{29}$. Similarly, optogenetic stimulations of interneurons led to LVFA whereas stimulation of pyramidal cells led to pre-ictal spiking in other 
studies 30,31 . Moreover, in intracellular recordings, initial LVFA is associated with tonic interneurons firing and transitory silencing of principal cells of both superficial and depth layers ${ }^{32}$.

A neuronal-population based model also confirmed the crucial role of interneuron inhibition on pyramidal cells in the transition from interictal to ictal state. In this model, a progressive decrease of dendritic inhibition followed by a decrease of somatic inhibition (with constant excitation) led to pre-ictal spiking followed by LVFA seizureonset pattern ${ }^{33}$. We could hypothesize that the SOP depends on the underlying excitatory/inhibitory balance and the micro-connectivity alteration. Therefore, one hypothesis is that in FCD some particular physiopathological alterations exist, including perhaps altered balance between interneurons and pyramidal cells leading to a specific SOP, namely bursts of polyspikes. Experimental studies have shown specific alteration of the population of interneurons in FCD both quantitatively and qualitatively 34, supporting this hypothesis. Nevertheless, further studies using specific animal model of FCD are required to confirm this.

This finding is also of particular clinical interest in patients with negative or unclear MRI because in this case the observation of a burst of polyspikes followed by a LVFA is strongly suggestive of FCD, classically associated with better post-surgical seizure outcome ${ }^{35}$. Moreover, knowing that some intracranial SOP have correlates on scalpEEG in case of superficial lesions ${ }^{36}$, their observation during non-invasive pre-surgical work-up could suggest certain underlying etiology.

\section{The EZ organization is a significant determinant of the SOP}

In a model developed by our group, the seizure onset occurs in a set of brain regions (the EZ) before disseminating to other specific regions (the propagation zone) ${ }^{37}$. SOP 
takes place in the EZ and we found that network organization of the EZ was the most frequent pattern of organization $(\sim 60 \%)$. This result agrees with previous reports 37 . Few studies have specifically investigated the relation between the extension of the EZ and the SOP. Some have shown a tendency of wider EZ in case of LVFA $4,9,15,38$, while others have failed to demonstrate association between EZ organization and SOP5 . Therefore, our study brings important information showing that the presence of a DC shift / slow-wave prior to LVFA was more frequently associated with a network EZ organization. One hypothesis could be that high amplitude slow-wave/baseline-shift before LVFA helps to synchronize distant areas within the EZ.

The effect of the organization of the EZ was previously suggested in mesio-temporal epilepsy with a difference in coupling between epileptogenic structures according to SOP $^{8}$. The neuronal-population based model emphasized also the importance of connectivity between neuronal population showing the influence of the coupling and its directionality in the genesis of $\mathrm{SOP}^{39}$. Nevertheless, to our knowledge no experimental study has specifically investigated this question.

\section{$\underline{\text { SOP is associated with post-surgical seizure outcome }}$}

We also showed that SOP is associated with post-surgical outcome independently of the extension of the EZ. Our findings confirm better prognosis in patterns including LVFA and conversely poorer prognosis in patients with rhythmic slow spikes or sharp theta/alpha. Most previous studies have already suggested better prognosis in case of LVFA $10,38,40-45$. We highlight the very good prognostic value of bursts of polyspikes prior to LVFA with $80 \%$ of patients becoming seizure-free, a proportion twice as high as for cases without LVFA, probably because this SOP indicated the presence of FCD with focal EZ organization. 
$36 \%$ of patients with slow seizure-onset patterns (rhythmic slow wave or sharp theta/alpha) became seizure-free after surgery in our study. This percentage is in accordance with previous studies reporting fewer patients $13,38,40,46,47$. The fact that about $1 / 3$ of patients became seizure-free after a resection based on SOP not including LVFA, argues against the notion that slow SOP are solely propagation patterns. Another argument is that these patterns could also be observed within epileptogenic lesions (MCD) ${ }^{5}$. Therefore, patients exhibiting these slow patterns should not be surgically contraindicated on the sole suspicion of a propagation pattern having been recorded. We also highlight the modest statistical effect of the SOP compared with the extension of the EZ (defined as focal or network). Similar findings have been reported concerning the extension of the EZ 12,40,43,45. Moreover, the completeness of the resection of the EZ (crucially linked with its rapports with eloquent area) is another essential factor in order to guarantee seizure-freedom $35,48-50$. While seizure onset pattern was independently associated with surgical prognosis, its predictive value in comparison with other important factors (completeness of EZ resection, EZ organization) cannot be fully extrapolated from our data. Further study looking at seizure onset patterns (and potentially other electrographic features) in a homogenous prospective cohort of patients, is required to assess their predictive power as biomarker of surgical prognosis.

\section{Limitations}

There are some limitations in our study, notably that we could had underestimated the number of network-organized EZ because of our restricted definition and the relatively limited number of electrodes per patients. Another limitation concerned our hardware high-pass filter at $0.16 \mathrm{~Hz}$ that could have limited our ability to detect DC shifts in some patients. 


\section{Conclusion}

In this study, we demonstrated in a large series of patients explored by SEEG with various causes of epilepsies that SOP is strongly dependent on underlying etiology and EZ organization. The main determinant factor of post-surgical seizure outcome is spatial organization of the EZ, but the SOP plays also a role with better prognosis when LVFA occurs. 


\section{ACKNOWLEGEMENTS}

We thank Prof. Patrick Chauvel, Prof. Martine Gavaret, Dr. Francesca Bonini, Dr. Lisa Vaugier, Dr. Sandrine Aubert, Dr. Geraldine Daquin, Dr. Constanza Dalvit, Dr. Nathalie Villeneuve and Dr. Anne Lepine (Marseille), for the clinical management of some included patients. We thank Prof. Henry Dufour and Prof. Jean-Claude Peragut (Marseille), Prof Jean Régis for surgical procedures in some of the selected patients.

\section{FUNDING}

This work has been carried out within the FHU EPINEXT with the support of the A*MIDEX project (ANR-11-IDEX-0001-02) funded by the "Investissements d'Avenir" French Governement program managed by the French National Research Agency (ANR). Part of this work was funded by a joint Agence Nationale de la Recherche (ANR) and Direction Génerale de l'Offre de Santé (DGOS) under grant "VIBRATIONS" ANR-13PRTS-0011-01.

\section{DISCLOSURE OF CONFLICTS OF INTEREST}


None of the authors has any conflict of interest to disclose. We confirm that we have read the Journal's position on issues involved in ethical publication and affirm that this report is consistent with those guidelines.

\section{REFERENCES}

1. Isnard J, Taussig D, Bartolomei F, et al. French guidelines on stereoelectroencephalography (SEEG). Neurophysiol Clin. 2017; .

2. Bartolomei F, Nica A, Valenti-Hirsch MP, et al. Interpretation of SEEG recordings. Neurophysiol Clin. 2017; .

3. Singh S, Sandy S, Wiebe S. Ictal onset on intracranial EEG: Do we know it when we see it? State of the evidence. Epilepsia. 2015; 56:1629-38.

4. Perucca P, Dubeau F, Gotman J. Intracranial electroencephalographic seizureonset patterns: Effect of underlying pathology. Brain. 2014; 137:183-96.

5. Lagarde S, Bonini F, McGonigal A, et al. Seizure-onset patterns in focal cortical dysplasia and neurodevelopmental tumors: Relationship with surgical prognosis and neuropathologic subtypes. Epilepsia. 2016; 57:1426-35.

6. Avoli M, de Curtis M, Gnatkovsky V, et al. Specific imbalance of excitatory/inhibitory signaling establishes seizure onset pattern in temporal lobe epilepsy. J Neurophysiol. 2016; 115:3229-37. 
7. Courtens S, Colombet B, Trébuchon A, et al. Graph Measures of Node Strength for Characterizing Preictal Synchrony in Partial Epilepsy. Brain Connect. 2016; 6:5309.

8. Bartolomei F, Wendling F, Régis J, et al. Pre-ictal synchronicity in limbic networks of mesial temporal lobe epilepsy. Epilepsy Res. 2004; 61:89-104.

9. Velascol AL, Wilson CL, Babb TL, et al. Functional and Anatomic Correlates of Two Frequently Observed Temporal Lobe Seizure-Onset Patterns. Neural Plast. 2000; $7: 49-63$.

10. Spanedda F, Cendes F, Gotman J. Relations between EEG seizure morphology, interhemispheric spread, and mesial temporal atrophy in bitemporal epilepsy. Epilepsia. 1997; 38:1300-14.

11. Schuh L a, Henry TR, Ross D a, et al. Ictal spiking patterns recorded from temporal depth electrodes predict good outcome after anterior temporal lobectomy. Epilepsia. 2000; 41:316-9.

12. Park YD, Murro a M, King DW, et al. The significance of ictal depth EEG patterns in patients with temporal lobe epilepsy. Electroencephalogr Clin Neurophysiol. 1996; 99:412-5.

13. Wetjen NM, Marsh WR, Meyer FB, et al. Intracranial electroencephalography seizure onset patterns and surgical outcomes in nonlesional extratemporal epilepsy. J Neurosurg. 2009; 110:1147-52.

14. Frauscher B, von Ellenrieder N, Dubeau F, et al. Different seizure-onset patterns in mesiotemporal lobe epilepsy have a distinct interictal signature. Clin Neurophysiol. 2017; 128:1282-9.

15. Weinand ME, Wyler AR, Richey ET, et al. Long-term ictal monitoring with subdural strip electrodes: prognostic factors for selecting temporal lobectomy candidates. J 
Neurosurg. 1992; 77:20-8.

16. Jayakar P, Gotman J, Harvey AS, et al. Diagnostic utility of invasive EEG for epilepsy surgery: Indications, modalities, and techniques. Epilepsia. 2016; $57: 1735-47$.

17. Blümcke I, Thom M, Aronica E, et al. International consensus classification of hippocampal sclerosis in temporal lobe epilepsy: A Task Force report from the ILAE Commission on Diagnostic Methods. Epilepsia. 2013; 54:1315-29.

18. Blümcke I, Thom M, Aronica E, et al. The clinicopathologic spectrum of focal cortical dysplasias: A consensus classification proposed by an ad hoc Task Force of the ILAE Diagnostic Methods Commission. Epilepsia. 2011; 52:158-74.

19. Louis DN, Perry A, Reifenberger G, et al. The 2016 World Health Organization Classification of Tumors of the Central Nervous System: a summary. Acta Neuropathol. 2016; 131:803-20.

20. Bartolomei F, Chauvel P, Wendling F. Epileptogenicity of brain structures in human temporal lobe epilepsy: A quantified study from intracerebral EEG. Brain. 2008; 131:1818-30.

21. Medina Villalon S, Paz R, Roehri N, et al. EpiTools, A software suite for presurgical brain mapping in epilepsy: Intracerebral EEG. J Neurosci Methods. 2018; 303:715.

22. Bartolomei F, Cosandier-Rimele D, McGonigal A, et al. From mesial temporal lobe to temporoperisylvian seizures: A quantified study of temporal lobe seizure networks. Epilepsia. 2010; 51:2147-58.

23. Bonini F, McGonigal A, Trebuchon A, et al. Frontal lobe seizures: From clinical semiology to localization. Epilepsia. 2014; 55:264-77.

24. Bartolomei F, Gavaret M, Hewett R, et al. Neural networks underlying parietal lobe 
seizures: A quantified study from intracerebral recordings. Epilepsy Res. 2011; 93:164-76.

25. Marchi A, Bonini F, Lagarde S, et al. Occipital and occipital "plus" epilepsies: A study of involved epileptogenic networks through SEEG quantification. Epilepsy Behav. 2016; 62:104-14.

26. Engel J. Update on surgical treatment of the epilepsies. Summary of the Second International Palm Desert Conference on the Surgical Treatment of the Epilepsies (1992). Neurology. 1993; 43:1612-7.

27. Nagelkerke NJD. A note on a general definition of the coefficient of determination. Biometrika. 1991.

28. Cox DR, Snell EJ. Analysis of binary data. London: Chapman and Hall. 1989.

29. Bragin A, Azizyan A, Almajano J, et al. The Cause of the Imbalance in the Neuronal Network Leading to Seizure Activity Can Be Predicted by the Electrographic Pattern of the Seizure Onset. J Neurosci. 2009; 29:3660-71.

30. Shiri Z, Manseau F, Lévesque M, et al. Interneuron activity leads to initiation of low-voltage fast-onset seizures. Ann Neurol. 2015; 77:541-6.

31. Shiri Z, Manseau F, Lévesque M, et al. Activation of specific neuronal networks leads to different seizure onset types. Ann Neurol. 2016; 79:354-65.

32. Gnatkovsky V, Librizzi L, Trombin F, et al. Fast activity at seizure onset is mediated by inhibitory circuits in the entorhinal cortex in vitro. Ann Neurol. 2008; $64: 674-86$.

33. Wendling F, Bartolomei F, Bellanger JJ, et al. Epileptic fast activity can be explained by a model of impaired GABAergic dendritic inhibition. Eur J Neurosci. $2002 ; 15: 1499-508$.

34. Medici V, Rossini L, Deleo F, et al. Different parvalbumin and GABA expression in 
human epileptogenic focal cortical dysplasia. Epilepsia. 2016; 57:1109-19.

35. Fauser S, Essang C, Altenmüller D-M, et al. Long-term seizure outcome in 211 patients with focal cortical dysplasia. Epilepsia. 2015; 56:66-76.

36. Tanaka H, Khoo HM, Dubeau F, et al. Association between scalp and intracerebral electroencephalographic seizure-onset patterns: A study in different lesional pathological substrates. Epilepsia. 2018; 59:420-30.

37. Bartolomei F, Lagarde S, Wendling F, et al. Defining epileptogenic networks: Contribution of SEEG and signal analysis. Epilepsia. 2017; 58:1131-47.

38. Lee S-A, Spencer DD, Spencer SS. Intracranial EEG Seizure-Onset Patterns in Neocortical Epilepsy. Epilepsia. 2000; 1:297-307.

39. Wendling F, Bellanger JJ, Bartolomei F, et al. Relevance of nonlinear lumpedparameter models in the analysis of depth-EEG epileptic signals. Biol Cybern. 2000; 83:367-78.

40. Jung WY, Pacia S V, Devinsky R. Neocortical temporal lobe epilepsy: intracranial EEG features and surgical outcome. J Clin Neurophysiol. 1999; 16:419-25.

41. Wennberg R, Arruda F, Quesney LF, et al. Preeminence of extrahippocampal structures in the generation of mesial temporal seizures: Evidence from human depth electrode recordings. Epilepsia. 2002; 43:716-26.

42. Doležalová I, Brázdil M, Hermanová M, et al. Intracranial EEG seizure onset patterns in unilateral temporal lobe epilepsy and their relationship to other variables. Clin Neurophysiol. 2013; 124:1095-105.

43. Holtkamp M, Sharan A, Sperling MR. Intracranial EEG in predicting surgical outcome in frontal lobe epilepsy. Epilepsia. 2012; 53:1739-45.

44. Kim DW, Kim HK, Lee SK, et al. Extent of neocortical resection and surgical outcome of epilepsy: Intracranial EEG analysis. Epilepsia. 2010; 51:1010-7. 
45. Faught E, Kuzniecky RI, Hurst DC. Ictal EEG wave forms from epidural electrodes predictive of seizure control after temporal lobectomy. Electroencephalogr Clin Neurophysiol. 1992; 83:229-35.

46. Kutsy RL, Farrell DF, Ojemann G a. Ictal patterns of neocortical seizures monitored with intracranial electrodes: correlation with surgical outcome. Epilepsia. 1999; 40:257-66.

47. Jiménez-Jiménez D, Nekkare R, Flores L, et al. Prognostic value of intracranial seizure onset patterns for surgical outcome of the treatment of epilepsy. Clin Neurophysiol. 2015; 126:257-67.

48. Chassoux F, Devaux B, Landre E, et al. Stereoelectroencephalography in focal cortical dysplasia: A 3D approach to delineating the dysplastic cortex. Brain. 2000; $123: 1733-51$.

49. Tassi L, Colombo N, Garbelli R, et al. Focal cortical dysplasia: neuropathological subtypes, EEG, neuroimaging and surgical outcome. Brain. 2002; 125:1719-32.

50. Bonini F, McGonigal A, Scavarda D, et al. Predictive Factors of Surgical Outcome in Frontal Lobe Epilepsy Explored with Stereoelectroencephalography. Neurosurgery. 2017; 0:1-9. 


\section{FIGURES LEGENDS}

Figure 1: The eight Seizure Onset Patterns according to the time frequency representation from SEEG trace. A: Low-voltage fast activity (LVFA); B: Preictal spiking followed by LVFA; C: Burst of polyspikes followed by LVFA; D: Slow wave or baseline shift followed by LVFA; E: Rhythmic slow spikes; F: Theta/alpha sharp activity; G: beta sharp activity; H: delta-brush.

The red asterisks mark the seizure onset.

Figure 2: Circle chart showing the overall prevalence of the eight Seizure Onset Patterns. Note that patterns without low voltage fast activity was found in about $30 \%$ of the patients.

Figure 3: Histogram showing the repartition of the Seizure Onset Pattern according to the etiology of the epilepsy. MCD: malformation of cortical development, NDT: neurodevelopmental tumors. Numbers indicates the number of patients per subgroup. Figure 4: Histogram showing the repartition of the Seizure Onset Pattern according to the presence or not of a lesion on the MRI. Numbers indicates the number of patients 
per subgroup.

Figure 5: Histogram showing the repartition of the Seizure Onset Pattern according to the spatial organization of the epileptogenic zone (EZ): focal or network. Numbers indicates the number of patients per subgroup.

Figure 6: Histogram showing the repartition of the post-surgical seizure outcome (seizure-free or not) according to the Seizure Onset Patterns. LVFA: low voltage fast activity. Numbers indicates the number of patients per subgroup. Mean follow-up duration $=69.6$ months . 